\title{
Oxygen desaturation during a 6 min walk test is a sign of nocturnal hypoxemia
}

\author{
Adrienne S Scott MS ${ }^{1}$, Marcel A Baltzan MD FRCPC ${ }^{1}$, Ryan Chan BSc ${ }^{2}$, Norman Wolkove MD FRCPC FCCP1
}

\author{
AS Scott, MA Baltzan, R Chan, N Wolkove. Oxygen desaturation \\ during a 6 min walk test is a sign of nocturnal hypoxemia. Can \\ Respir J 2011;18(6):333-337.
}

BACKGROUND/OBJECTIVES: Patients with chronic obstructive pulmonary disease (COPD) may experience sleep disordered breathing with nocturnal desaturation. An exploratory study was performed to determine whether any commonly measured clinical parameters were useful in predicting nocturnal desaturation in patients with COPD. A validation study was subsequently performed to confirm the utility of the parameter identified in the exploratory study as most useful in this regard.

METHODS: A total of 103 (exploratory cohort) and 200 (validation cohort) consecutive patients with COPD admitted for pulmonary rehabilitation were evaluated. Standard outcome measures including nocturnal oximetry and the 6 min walk test (6MWT) on room air with continuous pulse oximetry were assessed. Patients with sleep apnea or those undergoing long-term oxygen therapy were excluded.

RESULTS: In the exploratory study, the mean $( \pm$ SD) patient age was $70 \pm 9.9$ years, with forced expiratory volume in $1 \mathrm{~s}$ of $0.76 \pm 0.34 \mathrm{~L}$, which was $36 \pm 16 \%$ of predicted. Body mass index, arterial oxygen tension, oxygen saturation by pulse oximetry at rest and during the 6MWT all demonstrated significant correlations with percentage of time spent with a saturation $<90 \%$. When the lowest pulse oximetry during the $6 \mathrm{MWT}$ was $\leq 88 \%, 10$ of 21 patients demonstrated a saturation $<90 \%$ for at least $30 \%$ of sleep time. This measure yielded a positive likelihood ratio of 3.77 (95\% CI 1.87 to 7.62) compared with those who did not reach this threshold value. The validation study confirmed similar detection characteristics.

CONCLUSIONS: Results from the present study suggest that monitoring oxygen saturation changes during a 6MWT is useful in helping to identify COPD patients who may experience significant nocturnal desaturation.

Key Words: 6 min walk test; Chronic obstructive pulmonary disease; Exercise; Nocturnal hypoxemia

Some patients with chronic obstructive pulmonary disease (COPD) $\checkmark$ experience significant hypoxemia. For those with a resting partial pressure of oxygen in arterial blood $\left(\mathrm{PaO}_{2}\right)<55 \mathrm{mmHg}$, supplemental oxygen administration has been shown to be beneficial, particularly in improving exercise tolerance and survival $(1,2)$. However, less well recognized is that some individuals with COPD may desaturate only during sleep and become substantially more hypoxemic at night than they are during the day. Although nocturnal oximetry can readily identify such individuals, this requires overnight monitoring and is time consuming. Furthermore, it is unclear which patients require such a study. It would be useful if simple daytime parameters could help predict which patients are more likely to exhibit significant nocturnal desaturation (SND). In the present study, we sought to determine whether any parameters routinely measured in patients with COPD could be helpful in predicting the occurrence of significant sleeprelated oxygen desaturation. In particular, we aimed to determine whether measuring arterial oxygen saturation $\left(\mathrm{SpO}_{2}\right)$ during a 6 min walk test $(6 \mathrm{MWT})$ could provide additional information that might enable us to predict which patients were more likely to experience SND. The main finding from an initial study was applied to a second population to confirm its predictive capacity for the identification of patients who experience SND.

\section{Une désaturation en oxygène pendant un test de marche de 6 minutes est un signe d'hypoxémie nocturne}

HISTORIQUE ET OBJECTIFS : Les patients atteints d'une maladie pulmonaire obstructive chronique (MPOC) peuvent présenter des troubles respiratoires du sommeil accompagnés d'une désaturation nocturne. Les chercheurs ont mené une étude exploratoire pour déterminer si des paramètres cliniques souvent mesurés étaient utiles pour prédire une désaturation nocturne chez les patients atteints d'une MPOC. Ils ont ensuite procédé à une étude de validation pour confirmer que le paramètre repéré dans l'étude exploratoire était bien le plus utile à cet égard.

MÉTHODOLOGIE : Au total, 103 (cohorte d'exploration) et 200 (cohorte de validation) patients consécutifs atteints d'une MPOC admis en réadaptation pulmonaire ont été évalués. Les chercheurs ont évalué les mesures d'issue standards, y compris la saturométrie nocturne et le test de marche de 6 minutes (TM6M) à l'air ambiant surveillé par saturométrie continue. Les patients faisant de l'apnée du sommeil ou sous oxygénothérapie à long terme étaient exclus.

RÉSULTATS : Pendant l'étude exploratoire, les patients avaient un âge moyen $( \pm E ́ T)$ de $70 \pm 9,9$ ans, un volume expiratoire maximal par seconde de $0,76 \pm 0,34 \mathrm{~L}$, soit $36 \pm 16 \%$ des prévisions. L'indice de masse corporelle, la tension du sang artériel en oxygène ainsi que la saturométrie au repos et pendant le TM6M ont tous démontré des corrélations significatives avec le pourcentage de temps passé sous saturation inférieure à $90 \%$. Lorsque la saturométrie la plus basse pendant le TM6M était égale ou inférieure à $88 \%$, dix des 21 patients présentaient une saturation inférieure à $90 \%$ pendant au moins $30 \%$ de la période de sommeil. Cette mesure a suscité un rapport de vraisemblance positif de 3,77 (95\% IC1,87 à 7,62) par rapport aux patients qui n'avaient pas atteint cette valeur seuil. L'étude de validation a confirmé des caractéristiques de détection similaires.

CONCLUSIONS : Selon les résultats de la présente étude, la surveillance des modifications de la saturation en oxygène pendant le TM6M peut contribuer à dépister les patients atteints d'une MPOC qui peuvent présenter une importante désaturation nocturne.

\section{Study population}

\section{METHODS}

The data for the exploratory study were obtained by conducting a chart review of patients with a history of COPD who had been admitted for pulmonary rehabilitation at Mount Sinai Hospital (Montreal, Quebec) between March 2006 and May 2007. Subjects in the validation study were selected retrospectively (July 2008 and July 2009) based on results from a 6MWT in which they desaturated to or below $88 \%$. Approximately two age-matched controls were included per subject. The control patients had COPD but did not desaturate to or below $88 \%$. Patients with a history of obstructive sleep apnea were excluded, as were individuals who were on long-term supplemental oxygen. The present study was approved by the Mount Sinai Hospital Center Ethics Committee (approval number 052007).

\section{Study design}

All patients underwent routine spirometry that was performed according to published guidelines (3). Arterial blood gases were not analyzed routinely but were performed at the discretion of the attending physician. A 6MWT was conducted before initiation of the rehabilitation program using published recommendations (4). This was performed in a hospital corridor, and the total distance travelled was recorded in

${ }^{1}$ Reasearch Department, Mount Sinai Hospital Center; ${ }^{2}$ Faculty of Medicine, School of Physical and Occupational Therapy, McGill University,

Montreal, Quebec

Correspondence and reprints: Dr Norman Wolkove, Mount Sinai Hospital Center, 5690 Cavendish Boulevard, Cote St-Luc, Montreal,

Quebec H4W 1S7. Telephone 514-369-2222 ext 1060, fax 514-369-2225, e-mail nwolkove.sinai@ssss.gouv.qc.ca; marc.baltzan@clinepi.mcgill.ca 


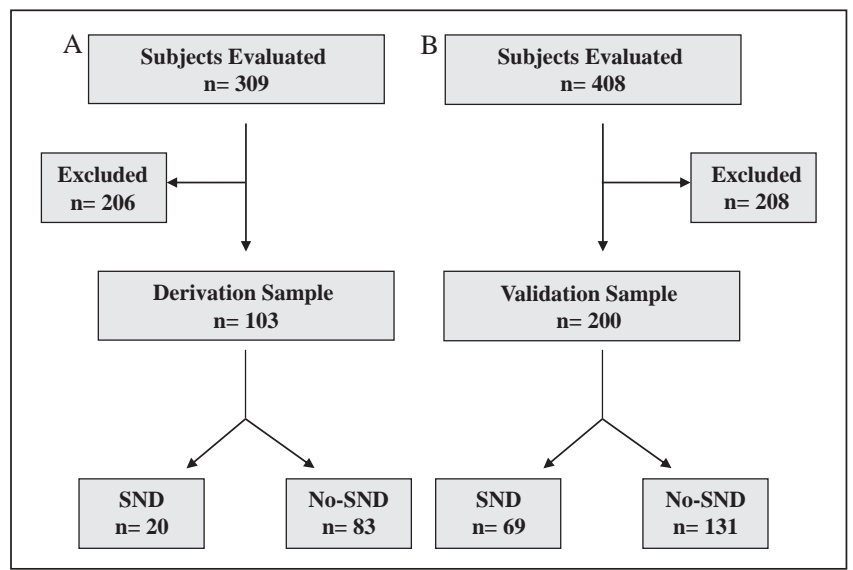

Figure 1) Study flow diagram A Exploratory study. B Validation cohort. SND Significant nocturnal desaturation

TABLE 1

Baseline characteristics of the exploratory and validation cohorts

\begin{tabular}{|c|c|c|}
\hline \multirow[b]{2}{*}{ Characteristic } & \multicolumn{2}{|c|}{ Cohort } \\
\hline & Exploratory $(n=103)$ & Validation $(n=200)$ \\
\hline Age, years & $70.1 \pm 9.9$ & $69.9 \pm 9.9$ \\
\hline Men, n (\%) & $47(46)$ & $94(47)$ \\
\hline Body mass index, $\mathrm{kg} / \mathrm{m}^{2}$ & $25.6 \pm 7.1$ & $25.1 \pm 6.2$ \\
\hline $\mathrm{FEV}_{1}, \mathrm{~L}$ & $0.76 \pm 0.34$ & $0.89 \pm 0.44$ \\
\hline $\mathrm{FEV}_{1}, \%$ predicted & $36.4 \pm 16.4$ & $41.7 \pm 21.7$ \\
\hline FVC, L & $1.8 \pm 0.5$ & $1.8 \pm 0.62$ \\
\hline FVC, \% predicted & $61 \pm 16$ & $57 \pm 19$ \\
\hline $\mathrm{FEV}_{1} / \mathrm{FVC}$ & $0.42 \pm 0.13$ & $0.49 \pm 0.15$ \\
\hline Arterial blood, $\mathrm{pH}$ & $7.4 \pm 0.03$ & $7.4 \pm 0.03^{\star}$ \\
\hline $\mathrm{PaCO}_{2}, \mathrm{mmHg}$ & $39.2 \pm 7.1$ & $41.0 \pm 6.4^{*}$ \\
\hline $\mathrm{PaO}_{2}, \mathrm{mmHg}$ & $69.6 \pm 8.0$ & $70.3 \pm 11.4^{*}$ \\
\hline 6 min walk distance, $\mathrm{m}$ & $176 \pm 71$ & $222 \pm 104$ \\
\hline
\end{tabular}

Data presented as mean $\pm S D$ unless otherwise indicated. *Arterial blood gas data were available for 81 (exploratory) and 137 (validation) patients. FEV 1 Forced expiratory volume in $1 \mathrm{~s}$; FVC Forced vital capacity; $\mathrm{PaCO}_{2}$ Partial pressure of carbon dioxide in arterial blood; $\mathrm{PaO}_{2}$ Partial pressure of oxygen in arterial blood

metres. The pulse oximetry saturation (ie, $\mathrm{SpO}_{2}$ ) was monitored using a finger sensor (Onyx II, Nonin Medical Inc, USA) and the values obtained were recorded before and immediately on completion of the 6MWT. If the patient stopped during the test, the $\mathrm{SpO}_{2}$ at that point was recorded. In addition, at these times, patients were asked to rate their perceived dyspnea on a 10-point Borg scale. During overnight oximetry, $\mathrm{SpO}_{2}$ was continuously recorded for a minimum of $6 \mathrm{~h}$ using a pulse oximeter and finger sensor (920M Plus oximeter, Respironics Inc, USA) while the patient slept in his/her hospital bed. A hard-copy tracing was then produced from which mean and lowest overnight saturation was obtained, as well as the percentage of valid recorded time spent at saturation $<90 \%$. As previously defined, a patient was characterized to have SND if $\geq 30 \%$ of overnight recorded time was spent with an oxygen saturation $<90 \%$ (5). Body mass index (BMI) was calculated by dividing body weight $(\mathrm{kg})$ by height $\left(\mathrm{m}^{2}\right)$.

\section{Statistical analysis}

Descriptive statistics (means, SDs, counts and frequencies in per cent) were used to describe patients' baseline characteristics. Pearson correlations were also calculated to evaluate the relationship between continuous baseline variables and percentage of time spent at $<90 \%$ saturation. A contingency table and ROC analysis was performed for the prediction of significant desaturation. Statistical significance was set at a two-sided level of 0.05 , and $95 \%$ CIs were calculated.
Table 2

6 min walk test results (exploratory study) $(n=103)$

\begin{tabular}{lc}
\hline Measurement & \\
\hline Prewalk $\mathrm{SpO}_{2}, \%$ & $93.9 \pm 2.4$ \\
Postwalk $\mathrm{SpO}_{2}, \%$ & $91.1 \pm 4.0$ \\
Change in oxygen saturation (prewalk - postwalk) & $2.8 \pm 3.1$ \\
6 min walk test distance, $\mathrm{m}$ & $175.8 \pm 70.9$ \\
Prewalk dyspnea score, 1-10 & $1.1 \pm 1.2$ \\
Postwalk dyspnea score, 1-10 & $3.4 \pm 2.1$ \\
\hline
\end{tabular}

Data presented as mean $\pm \mathrm{SD}$. $\mathrm{SpO}_{2}$ Oxygen saturation by pulse oximetry

TABLE 3

Overnight oximetry results (exploratory study) $(n=103)$

\begin{tabular}{lr}
\hline Measurement & \\
\hline Oxygen saturation, \% & $92.7 \pm 2.6$ \\
Highest oxygen saturation, \% & $98.3 \pm 1.4$ \\
Lowest oxygen saturation, \% & $73.5 \pm 14.9$ \\
Oxygen desaturation events <90\%, n & $105.0 \pm 126.0$ \\
Percentage of time at <90\% oxygen saturation & $14.4 \pm 21.9$ \\
\hline
\end{tabular}

Data presented as mean $\pm S D$

\section{RESULTS}

\section{Subjects}

The overall flow of participants and organization of the study protocols are summarized in Figure 1. The most common reasons for exclusion were an additional diagnosis of obstructive sleep apnea (45\%), use of oxygen (26\%) and insufficient data (29\%). The descriptive characteristics of both the exploratory and validation cohorts are presented in Table 1. Both populations contained a high proportion of patients who were classified as having severe COPD.

Outcomes in the 6MWT and nocturnal oximetry (exploratory study)

Results of the 6MWT are summarized in Table 2. Patients generally demonstrated a reduced walk distance for their age and moderate dyspnea on exertion. Nocturnal oximetry data are detailed in Table 3. Twenty of 103 patients (20\%) met the criteria for SND (Figure 1).

Correlation and contingency analysis (exploratory study) BMI, $\mathrm{PaO}_{2}$ and $\mathrm{SpO}_{2}$ before and after the $6 \mathrm{MWT}$ all demonstrated significant correlations with the percentage of time spent with $\mathrm{SpO}_{2}$ $<90 \%$ during sleep (Table 4). The prevalence of SND was $48 \%$ (10 of 21 ) when the postwalk saturation was $\leq 88 \%$ compared with $12 \%$ ( 10 of 82 ) when the postwalk saturation was $>88 \%$. The critical threshold of $88 \%$ for desaturation during the 6MWT was supported by ROC analysis (Figure 2). The area under the curve statistic was 0.68 (95\% CI 0.54 to 0.82 ). A positive likelihood ratio for SND was 3.77 (95\% CI 1.87 to 7.62 ) in patients who desaturated with $\mathrm{SpO}_{2}$ $\leq 88 \%$ (Table 5). Of the patients who maintained a saturation $\geq 95 \%$ during exercise, only $5 \%$ (two of 44) experienced SND. The greatest sum of sensitivity (50\%) and specificity (87\%) was at a pulse oximetry threshold of $\leq 88 \%$, also suggesting that this value represented the optimum threshold for detection (6). The threshold $\mathrm{SpO}_{2}$ of $\leq 88 \%$ during a $6 \mathrm{MWT}$ was retained as the primary predictor of SND to validate in a subsequent cohort.

Evaluation of the primary predictor (validation study) The 200 patients used in the validation analysis were obtained as shown in Figure 1, with their characteristics also noted in Table 1. Contingency analyses (Table 5) using the same cut-off of $88 \%$ derived in the exploratory study, proved to be operational and was still predictive of SND. The ROC analysis supported the use of the $\mathrm{SpO}_{2}$ threshold $\leq 88 \%$ as a predictor of SND (Figure 3). The area under the curve in the validation population was 0.73 (95\% CI 0.65 to 0.80 ). 
TABLE 4

Interitem correlations $(r)$ of patient variables and percentage of time with arterial oxygen saturation $<90 \%$

\begin{tabular}{|c|c|}
\hline Outcomes & $\mathbf{r}$ \\
\hline \multicolumn{2}{|l|}{ Pulmonary function } \\
\hline $\mathrm{FEV}_{1}, \mathrm{~L}$ & -0.05 \\
\hline $\mathrm{FEV}_{1}, \%$ predicted & -0.09 \\
\hline FVC, L & -0.07 \\
\hline $\mathrm{FEV}_{1} / \mathrm{FVC}, \%$ & -0.02 \\
\hline \multicolumn{2}{|l|}{ Arterial blood gas, $\mathrm{mmHg}$} \\
\hline $\mathrm{PaO}_{2}$ & $-0.34^{\star \star}$ \\
\hline $\mathrm{PaCO}_{2}$ & 0.13 \\
\hline \multicolumn{2}{|l|}{6 min walk test } \\
\hline Distance, m & 0.02 \\
\hline Prewalk $\mathrm{SpO}_{2}, \%$ & $-0.40^{\star *}$ \\
\hline Postwalk $\mathrm{SpO}_{2}, \%$ & $-0.37^{* *}$ \\
\hline Prewalk dyspnea score, 1-10 & -0.08 \\
\hline Postwalk dyspnea score, 1-10 & -0.08 \\
\hline Body mass index & $0.23^{\star}$ \\
\hline
\end{tabular}

${ }^{*} P<0.05 ;{ }^{*} P<0.01$. FEV 1 Forced expiratory volume in $1 \mathrm{~s} ;$ FVC Forced vital capacity; $\mathrm{PaCO}_{2}$ Partial pressure of carbon dioxide in arterial blood; $\mathrm{PaO}_{2}$ Partial pressure of oxygen in arterial blood; $\mathrm{SpO}_{2}$ Oxygen saturation by pulse oximetry

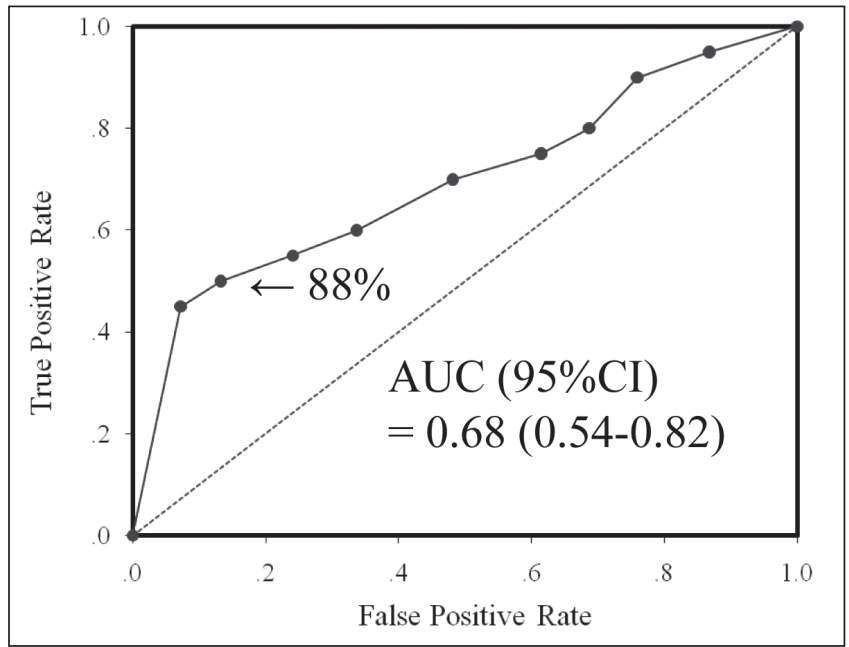

Figure 2) ROC curve. Pulse oximetry after the 6 min walk test in the validation cohort. AUC Area under the curve

\section{DISCUSSION}

In our initial (ie, exploratory) study, we found that desaturation following a 6MWT was useful in helping to predict which patients with COPD had SND. Almost one-half of subjects who had a 6MWT with a lowest $\mathrm{SpO}_{2} \leq 88 \%$ during their walk, had SND according to our definition. This represented a positive likelihood of 3.77 compared with those who did not reach this threshold value. The findings were consistent in our validation study, in which 36 of 63 (57\%) patients with a $6 \mathrm{MWT} \mathrm{SpO}_{2} \leq 88 \%$ had SND. Because the $6 \mathrm{MWT}$ is frequently used to assess patients, we suggest that $\mathrm{SpO}_{2}$ also be monitored during this evaluation. Using the above threshold level, individuals with the highest likelihood of having SND can be identified, thus enabling physicians to use overnight oximetry in a population in which the probability of finding SND is high.

We chose to focus particularly on the 6MWT because it has been established as an informative parameter in patients with $\operatorname{COPD}(7,8)$. The 6MWT distance has been shown to be a better predictor of mortality than forced expiratoy volume in $1 \mathrm{~s}\left(\mathrm{FEV}_{1}\right)$ in these individuals $(9,10)$. Casanova et al $(7)$ found that oxygen desaturation during the 6MWT can help predict which patients may experience a poor outcome independent from the distance walked. Garcia-Talavera et al (8) also advocated recording $\mathrm{SpO}_{2}$ during the 6MWT, and found that
TABLE 5

Diagnostic characteristics at different oxygen saturation thresholds achieved during the 6 min walk test

\begin{tabular}{llllc}
\hline Exploratory cohort & & \multicolumn{3}{c}{ Nocturnal desaturation } \\
\cline { 3 - 5 } & & Yes & No & Total \\
\hline Exercise-induced & Yes & 10 & 11 & 21 \\
desaturation & No & 10 & 72 & 82 \\
& Total & 20 & 83 & 103 \\
\hline
\end{tabular}

Data presented as n. Sensitivity: 10/20=50\%; Specificity: 72/83=87\%; Positive likelihood ratio $=3.77$

Validation cohort

\begin{tabular}{cllcr}
\hline & & \multicolumn{3}{c}{ Nocturnal desaturation } \\
\cline { 3 - 5 } Exercise-induced & & Yes & No & Total \\
\cline { 3 - 5 } desaturation & Yes & 36 & 27 & 63 \\
& No & 31 & 106 & 137 \\
& Total & 67 & 133 & 200 \\
\hline
\end{tabular}

Data presented as $n$. Sensitivity 36/67=54\%; Specificity: $106 / 133=80 \%$; Positive likelihood ratio $=2.65$

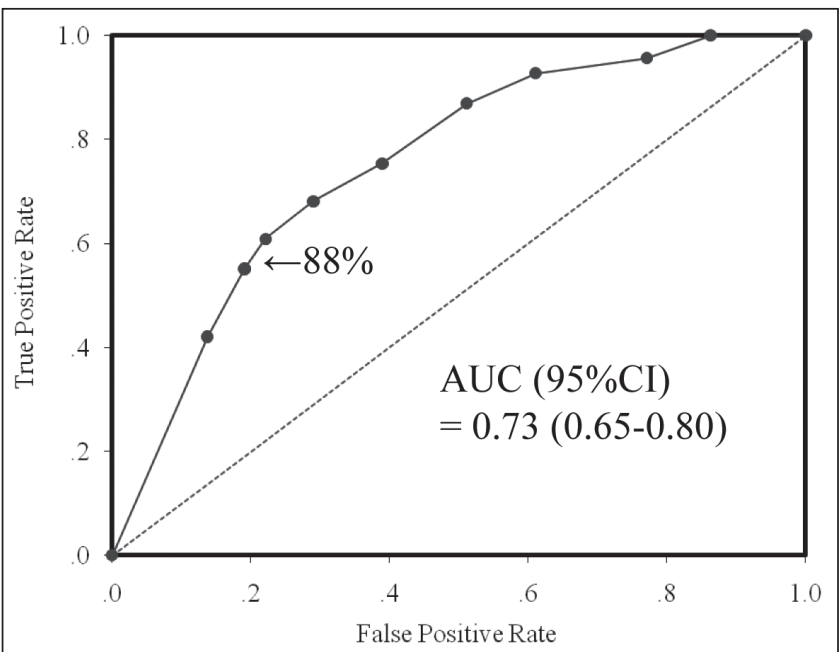

Figure 3) ROC curve. Pulse oximetry after the $6 \mathrm{~min}$ walk test in the validation cohort. AUC Area under the curve

patients with COPD who desaturated during the first minute of their walk were those most likely to desaturate during activities of daily living or during sleep.

Although we believed that desaturation during the 6MWT was most useful in predicting SND, we found that several other baseline measurements also correlated with nocturnal desaturation to levels $<90 \%$. These included a reduced $\mathrm{PaO}_{2}$ and/or $\mathrm{SpO}_{2}$ at rest before the $6 \mathrm{MWT}$. Nocturnal desaturation also correlated with BMI. These relationships have been noted by other authors, but their predictive value has been variable. Fletcher et al (11) found a low $\mathrm{PaO}_{2}$ and a high partial pressure of carbon dioxide in arterial blood $\left(\mathrm{PaCO}_{2}\right)$ to be related to - but not very predictive of - nocturnal desaturation. Bradley et al (12), however, identified awake $\mathrm{SpO}_{2}$ and $\mathrm{PaCO}_{2}$ as independent and predictive variables related to nocturnal desaturation. Similarly, Zanchet and Viegas (13) found that the only variable capable of predicting nocturnal desaturation was daytime $\mathrm{SpO}_{2}$. A limitation of these studies was their small sample size. Nevertheless, these studies and ours reinforce the intuitive notion that individuals with low baseline oxygen saturation are more likely to desaturate at night because they are already closer to whichever threshold for defining these events is used. In addition, because they are starting at a lower baseline level, they are near the steep portion of the oxygen dissociation curve, where small changes in $\mathrm{PaO}_{2}$ can lead to relatively large changes in $\mathrm{SpO}_{2}$. 
Baseline lung function was not useful in predicting nocturnal desaturation in our study, a finding consistent with the literature $(14,15)$. Heijdra et al (16) were an exception, finding that in addition to gas exchange, $\mathrm{FEV}_{1}$ and maximal inspiratory muscle strength correlated with nocturnal desaturation. Zanchet and Viegas (13) highlighted the difference between correlates and predictors of nocturnal desaturation in patients with COPD. Although they found that the $\mathrm{FEV}_{1} / \mathrm{FVC}$ ratio correlated positively and significantly with $\mathrm{SpO}_{2}$ during sleep, the only variable capable of predicting nocturnal desaturation was daytime $\mathrm{SpO}_{2}$.

The prevalence of nocturnal desaturation among patients with COPD is unknown. Obviously, the lower the baseline $\mathrm{SpO}_{2}$ in the population studied the higher the prevalence of SND is likely to be. Little et al (17) observed nocturnal desaturation in patients with $\mathrm{SpO}_{2}$ $\leq 93 \%$ but in none with $\mathrm{SpO}_{2} \geq 95 \%$. Others have refuted these results, reporting, for example, patients with daytime saturation as low as $91 \%$ or $92 \%$ who do not have SND (13). Although unusual, we found several patients in our series with daytime saturation $\geq 95 \%$ who did have nocturnal desaturation. In one of the earliest attempts to address this issue, Fletcher et al (18) found that $27 \%$ of patients with an awake $\mathrm{PaO}_{2}$ of at least $60 \mathrm{mmHg}$ experienced REM-associated desaturation. Subsequently, several small studies of patients with COPD reported the prevalence of SND to be approximately $50 \%$ in patients who had some degree of daytime hypoxemia $(13,14)$. Similarly, after screening more than 800 patients with COPD, Lewis et al (19) found nocturnal desaturation in 29 of 59 patients with a daytime $\mathrm{SpO}_{2}<95 \%$. They then assumed that the remaining large group with a daytime $\mathrm{SpO}_{2}$ $\geq 95 \%$ would not desaturate at night (which may not be accurate) and, thus, estimated the overall incidence of nocturnal desaturation to be $4.8 \%$. Our study of 103 patients represents one of the largest in the literature attempting to address the issue of nocturnal desaturation in individuals with COPD. It is also unique in that it is the largest unselected group to be studied in such a way. We found that 20 of 103 $(19.4 \%)$ patients in our study had SND. This figure, intermediate between the values of Thomas et al (14), Zanchet and Viegas (13) and the conservative estimate of Lewis et al (19), probably reflects the fact that we included a wide range of individuals with COPD. In our study, for example, approximately $43 \%(n=44)$ of our patients demonstrated a daytime $\mathrm{SpO}_{2} \geq 95 \%$ at one extreme, while another $28 \%(\mathrm{n}=29)$ had a baseline $\mathrm{SpO}_{2} \leq 92 \%$.

Several mechanisms have been postulated to explain the development of nocturnal desaturation in patients with COPD. These include increased bronchial tone alveolar hypoventilation, ventilation perfusion mismatch, increased upper airway resistance and diminished responses to hypoxia and hyperapnea $(20,21)$.

The significance of nocturnal desaturation in patients with COPD remains unclear. Although two major trials have proven that supplemental oxygen therapy can reduce mortality among patients with COPD and persistent hypoxemia, the evidence supporting a similar benefit for patients who desaturate only at night is less obvious $(1,2)$. It is known that transient episodes of significant hypoxemia can produce vasoconstriction, pulmonary hypertension and arrythmias $(22,23)$. However, it has not been firmly established that patients with isolated nocturnal desaturation necessarily develop sustained pulmonary hypertension and have an increased risk of mortality. In one of the few studies to address this issue, Fletcher et al (18) concluded that nocturnal desaturation in patients with COPD was associated with shorter survival. A trend toward increased survival in 35 oxygen-treated versus 38 nontreated subjects was noted, but a statistically significant difference would likely have required more subjects. In a review of available studies in the literature, Cranston et al (24) concluded that home oxygen therapy did not appear to improve survival in patients who experienced desaturation at night only. Similarly, although many patients with COPD report poor quality or interrupted sleep, the limited data in the literature have not shown that nocturnal desaturation is associated with impairment in quality of life, sleep quality or daytime function $(19,25)$. However, the paucity of information available in this regard suggests that larger studies are needed if we are to more completely assess the significance of isolated nocturnal desaturation in patients with COPD.

Presently, a three-year, multicentre, placebo-controlled, randomized trial of nocturnal oxygen therapy (CANOX) has been designed and started by Lacasse et al in Canada (26). Patients to be studied are those with COPD and nocturnal desaturation only. This study will hopefully provide meaningful data to determine whether nocturnal oxygen therapy provided for a period of three years decreases mortality, improves quality of life and/or delays the prescription of long-term oxygen therapy in these patients with COPD.

ACKNOWLEDGEMENTS: Author contributions: Ms Scott: contributed to design, study coordination, data collection, data analysis, results interpretation, literature review, manuscript writing and revisions. Dr Baltzan: contributed to design, data collection, data analysis, results interpretation, manuscript writing and revisions. Dr Wolkove: contributed to design, results interpretation, literature review, manuscript writing and revisions. Mr Chan: contributed to the collection of data and analysis.

FINANCIAL/NONFINANCIAL DISCLOSURES: The authors have reported to the Canadian Respiratory Journal that no potential conflicts of interest exist with any companies/organizations whose products or services may be discussed in this article.

Funded by the Mount Sinai Hospital Research Fund. Dr Norman Wolkove has no conflict of interest to disclose. Adrienne S Scott has no conflict of interest to disclose. Dr Marcel A Baltzan has no conflict of interest to disclose. Ryan Chan has no conflict of interest to disclose.

\section{REFERENCES}

1. Nocturnal Oxygen Therapy Trial group. Continuous or nocturnal oxygen therapy in hypoxemic chronic obstructive lung disease: A clinical trial. Ann Intern Med 1980;93:391-8.

2. Medical Research Council Party. Long-term domiciliary oxygen therapy in chronic hypoxic cor pulmonale complicating chronic bronchitis and emphysema. Lancet 1981;i:681-6.

3. Miller MR, Crapo R, Hankinson J, et al. General considerations for lung function testing. Eur Respir J 2005;26:153-61.

4. ATS Statement: Guidelines for the 6-minute walk test. Am J Respir Crit Care Med 2002;166:111-7.

5. Levi-Valensi P, Weitzenblum E, Rida Z, et al. Sleep-related oxygen desaturation and daytime pulmonary haemodynamics in COPD patients. Eur Respir J 1992;5:301-7.

6. Connell FA, Koepsell TD. Measures of gain in certainty from a diagnostic test. Am J Epidemiol 1985;121:744-53.

7. Casanova C, Cote C, Marin JM, et al. Distance and oxygen desaturation during the 6 -min walk test as predictors of long-term mortality in patients with COPD. Chest 2008;134:746-52.

8. Garcia-Talavera I, Garcia CH, Macario CC, de Torres JP, Celli BR, Aguirre-Jaime A. Time to desaturation in the 6-min walk distance test predicts 24-hour oximetry in COPD patients with a $\mathrm{PO}_{2}$ between 60 and $70 \mathrm{mmHg}$. Respir Med 2008;102:1026-32.

9. Martinez FJ, Foster G, Curtis JL, et al. NETT Research Group: Predictors of mortality in patients with emphysema and severe airflow obstruction. Am J Respir Crit Care Med 2006;173:1326-34.

10. Pinto-Plata VM, Cote C, Cabral H, Taylor J, Celli BR. The 6-min walk distance: Change over time and value as a predictor of survival in severe COPD. Eur Respir J 2004;23:28-33.

11. Fletcher EC, Miller J, Divine GW, Fletcher JG, Miller T. Nocturnal oxyhemoglobin desaturation in COPD patients with arterial oxygen tensions above $60 \mathrm{mmHg}$. Chest 1987;92:604-8.

12. Bradley TD, Martinez D, Rutherford R, et al. Physiological determinants of nocturnal arterial oxygenation in patients with obstructive sleep apnea. J Appl Physiol 1985;59:1364-8.

13. Zanchet RC, Viegas CA. Nocturnal desaturation: Predictors and the effect on sleep patterns in patients with chronic obstructive pulmonary disease and concomitant mild daytime hypoxemia. J Bras Pneumol 2006;32:207-12. 
14. Thomas VD, Kumar V, Gitanjali B. Predictors of nocturnal oxygen desaturation in chronic obstructive pulmonary disease in a South Indian population. J Postgard Med 2002;48:101-4.

15. Cormick W, Olson LG, Hensley MJ, Saunders NA. Nocturnal hypoxaemia and quality of sleep in patients with chronic obstructive lung disease. Thorax 1986;41:846-54.

16. Heijdra YF, Dekhuijzen PN, van Herwaarden CL, Folgering HT. Nocturnal saturation and respiratory muscle function in patients with chronic obstructive pulmonary disease. Thorax 1995;50:610-2.

17. Little SA, Elkholy MM, Chalmers, Farouk GW, Patel KR, Thomson NC. Predictors of nocturnal oxygen desaturation with COPD. Respir Med 1999;93:202-7.

18. Fletcher EC, Donner CF, Midgren B, et al. Survival in COPD patients with a daytime $\mathrm{PaO}_{2}$ greater than $60 \mathrm{~mm} \mathrm{Hg}$ with and without nocturnal oxyhemoglobin desaturation. Chest 1992;101:649-55.

19. Lewis CA, Fergusson W, Eaton T, Zeng I, Kolbe J. Isolated nocturnal desaturation in COPD: Prevalence and impact on quality of life and sleep. Thorax 2009;4:133-8.
20. Berthon-Jones M, Sullivan CE. Ventilatory and arousal responses to hypoxia in normal sleeping humans. Am Rev Respir Dis 1982;125:632-9.

21. Catterall JR, Calverley PM, MacNee W, et al. Mechanism of transient nocturnal hypoxemia in hypoxic chronic bronchitis and emphysema. J Appl Physiol 1985;59:1698-703.

22. Flick MR, Block AJ. Nocturnal vs diurnal cardiac arrhythmias in patients with chronic obstructive pulmonary disease. Chest 1979;75:8-11.

23. Chaouat A, Naeije R, Weitzenblum E. Pulmonary hypertension in COPD. Eur Respir J 2008;32:1371-85.

24. Cranston JM, Crockett AJ, sMoss JR, Alpers JH. Domicilliary oxygen for chronic obstructive pulmonary disease. Cochrane Database Syst Rev 2005;19(4):CD001744.

25. Krachman S, Minai OA, Scharf S. Sleep abnormalities and treatment in emphysema. Proc Am Thorac Soc 2008;5:536-42.

26. Multi-center randomized placebo-controlled trial of nocturnal oxygen therapy in chronic obstructive pulmonary disease. The Canadian Nocturnal Oxygen (CANOX) Trial. $<$ www.clinicaltrials.gov> (NCT01044628). 


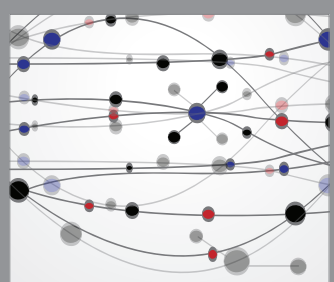

The Scientific World Journal
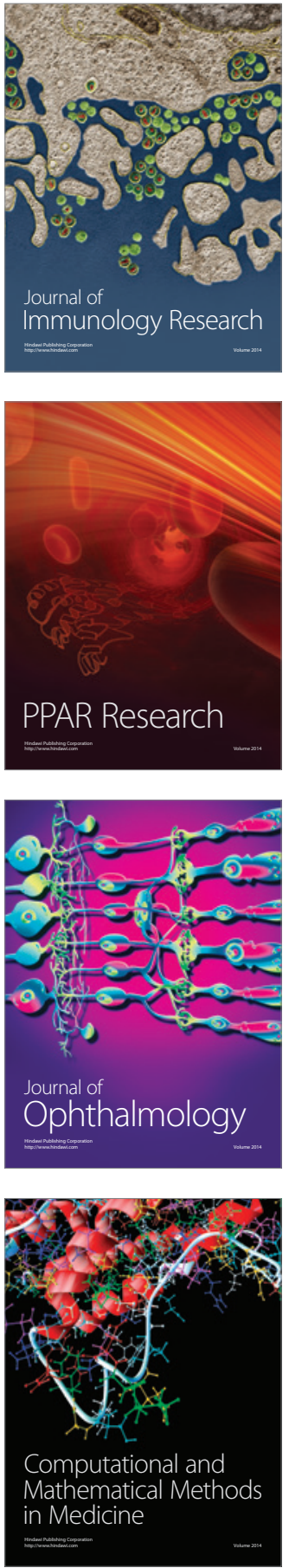

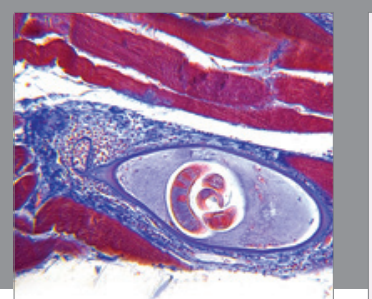

Gastroenterology Research and Practice

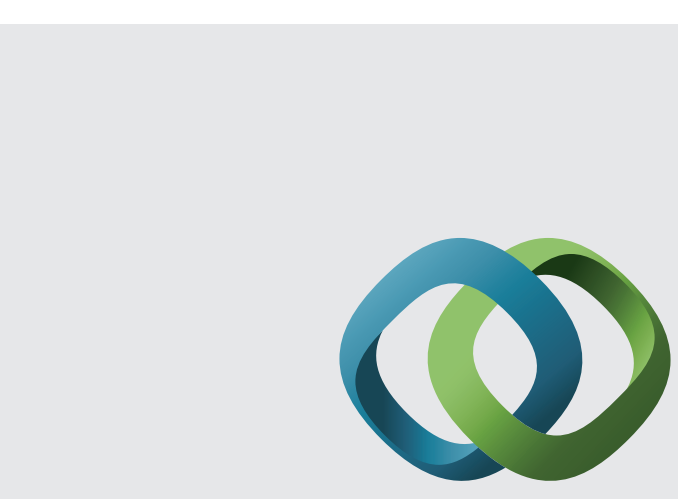

\section{Hindawi}

Submit your manuscripts at

http://www.hindawi.com
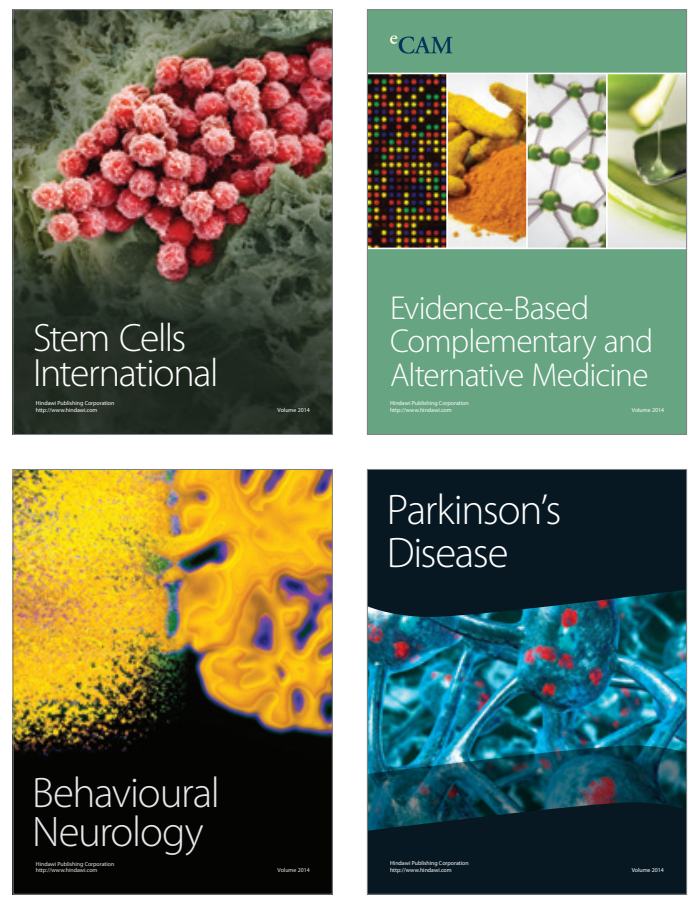
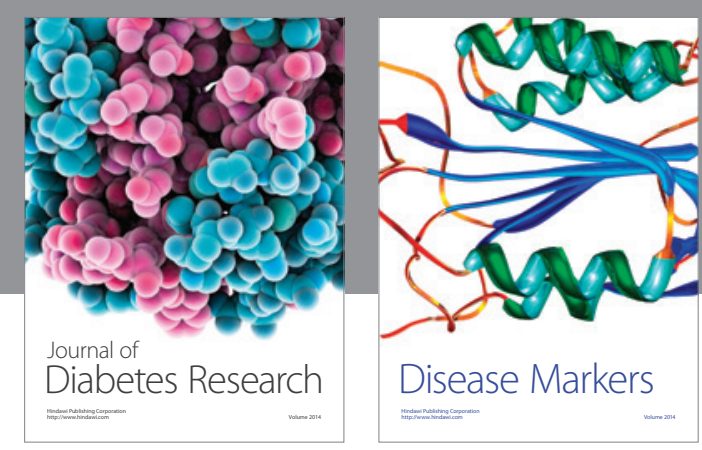

Disease Markers
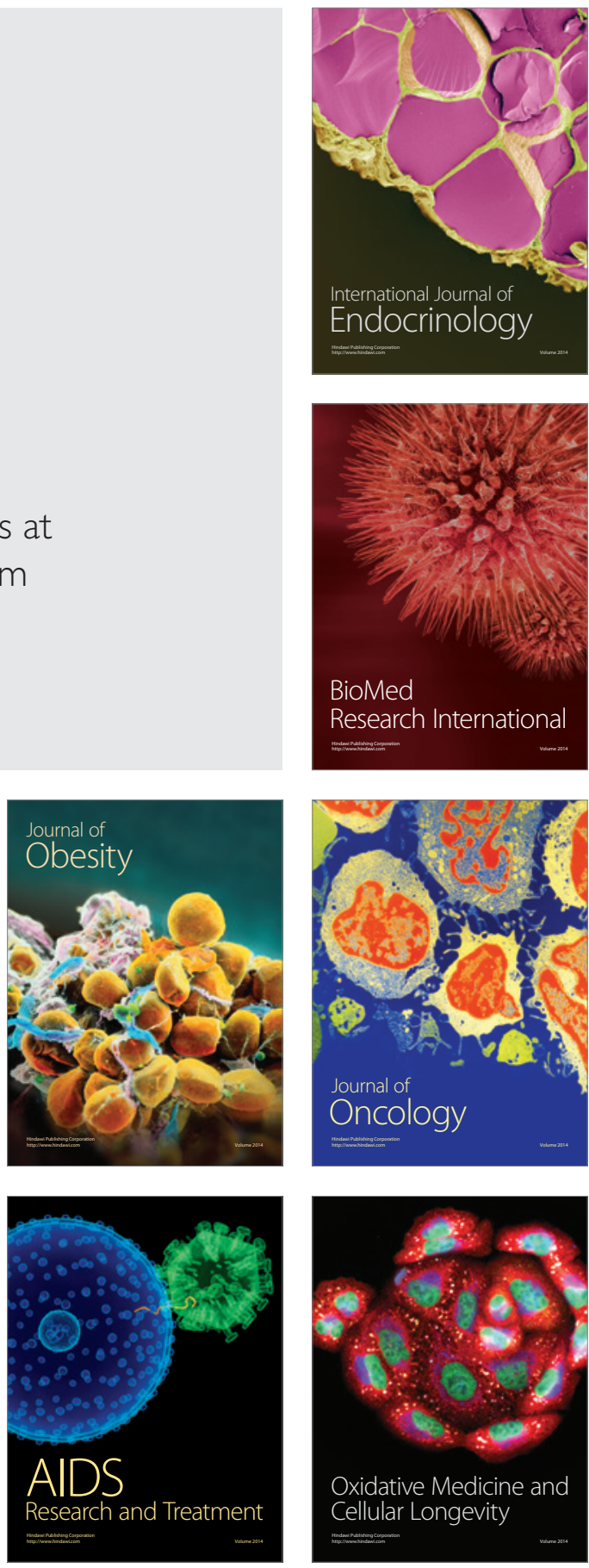